\title{
Epidemiología de la insuficiencia cardiaca: aproximación al paciente real en Galicia
}

\author{
Epidemiology of heart failure: The real patient in Galicia
}

\begin{abstract}
Julio Montes Santiago
Servicio de Medicina Interna. Hospital Meixoeiro. Complejo Hospitalario Universitario. Vigo. (CHUVI).
\end{abstract}

\section{Resumen}

OBJETIVO: Galicia es una de las Autonomía españolas con mayor tasa de mortalidad por insuficiencia cardiaca (IC). En los últimos años han sido elaborados diferentes perfiles de las poblaciones con IC en Galicia. En este trabajo realizamos una revisión de los datos disponibles sobre las características demográficas de la IC en dicha Comunidad.

MATERIAL Y MÉTODOS: Se estudiaron las características demográficas comunes en al menos 6 de los 7 siguientes estudios recientes: INCARGAL (951 pacientes), Hospital Clínico Universitario de Santiago (Card-CHUS)(1252 pacientes); GALICAP (1195 pacientes ingresados, seguidos posteriormente en Atención Primaria), GALICEP (25487 altas de Hospitales del SERGAS, periodo 2002-2005), EPICOUR (384 pacientes) o SOGAMI-IC (432 pacientes).

RESULTADOS: Excepto en pacientes ingresados en Servicios de Cardiología (Card-CHUS: 64), la media de edad estuvo en torno a los 78 años y más del $65 \%$ de pacientes fueron mayores de 75 años. En todos los estudios los antecedentes de HTA estuvieron presente en más de $2 / 3$ de los casos. Los días de estancia estuvieron en torno a los 11 días y la mortalidad hospitalaria en los Registros en torno al 7\% (INCARGAL) o al 10\% (GALICEP). Existe una elevada prevalencia de comorbilidad en las hospitalizaciones en tales pacientes.

CONCLUSIONES: Cerca de 2 de cada 3 pacientes con IC en Galicia son $>75$ años. Los pacientes ingresados en Cardiología son de menor edad y en mayor proporción varones. La mayoría de IC presentan HTA como antecedente 0 causa etiológica. Los días de estancia fueron cerca de 11 ías y se observó moderada letalidad mortalidad hospitalaria por IC (7-10\%). PALABRAS CLAVE: Insuficiencia cardiaca. Galicia.

\section{Introducción}

La insuficiencia cardiaca (IC) constituye una de las mayores causas de morbimortalidad en países occidentales. Así, se estima que entre el 0,4-2,0 de las poblaciones generales europeas y del $1,5-2 \%$ de las poblaciones norteamericanas y australianas padecen dicha entidad ${ }^{1-4}$. La insuficiencia cardiaca crónica (ICC) es la única entidad cardiovascular cuya incidencia ha continuado en aumento en países occidentales, hasta constituirse en una auténtica epidemia. Como acertadamente ha señalado un reciente editorial ${ }^{5}$ su desarrollo ejemplifica la conjunción de factores que han caracterizado a la moderna asistencia cardiovascular: la mejor detección y control de factores predisponentes tales como la hipertensión arterial (HTA) o la diabetes mellitus (DM), la mejoría en la supervivencia de la cardiopatía isquémica y otras enfermedades cardiovasculares -incluyendo la de la propia ICC-, junto a otros como el progresivo envejecimiento de la población o la mayor prevalencia de dichos factores de riesgo cardiovasculares como la propia HTA, la obesidad o el sedentarismo. Ello es particularmente evidente en el caso de la IC con fracción de

\begin{abstract}
OBJECTIVE: Galicia is one of the spanish autonomic community with increased mortality from heart failure (HF). In recent years different profiles of populations with HF in Galicia were studied. In this work we review the available data on the demographic characteristics of the IC in this community.

MATERIAL AND METHODS: We studied the demographic characteristics common to at least 6 of the following 7 recent studies: INCARGAL (951 patients), Hospital Clinico Universitario de Santiago (CHUS-Card) (1252 patients); GALICAP (1195 patients admitted, then followed by Primary carers), GALICEP (25487 discharges in SERGAS Hospitals, 2002-2005), EPICOUR (384 patients) or SOGAMI-IC (432 patients).

RESULTS: Except in patients admitted to Cardiology wards (Card-CHUS: 64), the median age was around 78 years old and $>65 \%$ of patients were older than 75 years. In all studies the history of hypertension were present in more than two thirds of cases. The length of stay was near around 11 days and in hospital mortality was in Registers around 7\% (INCARGAL) or 10\% in SERGAS (GALICEP). There was a high prevalence of comorbidity in these patients.

CONCLUSIONS: About 2 out of 3 patients with HF in Galicia are $>75$ years. Patients admitted to Cardiology are younger and in greater proportion males. Most HF have antecedent or a hipertensive etiology. The length of stay was about 11 days and a moderate lethality for HF was observed in hospitalizations (7-10\%).
\end{abstract}

KEYWORDS: Heart failure. Galicia.

eyección preservada (ICFEP). Esta entidad que ha recibido una atención importante en los últimos años pero en la que todavía subsisten notables puntos oscuros en lo referente a diagnóstico y tratamiento, presenta su mayor incidencia en mujeres y ancianos como trasunto claro de la mayor supervivencia poblacional hasta edades avanzadas y de la creciente incidencia en tales grupos demográficos de HTA, DM, fibrilación auricular (FA) y obesidad.

Tales expectativas de mayor longevidad y de mejoría de cuidados en las enfermedades cardiovasculares han llevado aparejadas, como contrapartida, un aumento de los ingresos hospitalarios por ICC y un incremento exponencial de sus costes atribuibles, del que dichas hospitalizaciones son responsables del $60-70 \%{ }^{1}$. Ello, en el contexto actual de crisis económica, ocasiona peligrosos desequilibrios en la viabilidad económica de los sistemas sanitarios. Además debe resaltarse que, a pesar de significativos avances producidos en la asistencia a esta entidad, la morbimortalidad de la ICC apenas parece haber descendido, en relación indudable con 
la mayor edad de la población y las frecuentes entidades comórbidas que complican este proceso $0^{1,2}$.

En este trabajo y antes de centrarnos en la situación específica de Galicia efectuaremos una panorámica de la situación mundial y española para mostrar la universalidad de este proceso y situar en la adecuada perspectiva la realidad en nuestro entorno.

\section{Magnitud global de la epidemia}

En la tabla 1 se ofrece una panorámica general de la situación mundial actual de la presente epidemia. Debe resaltarse que p.ej. que en EEUU actualmente se calcula en 6,6 millones los pacientes que padecen dicha epidemia, pero que alcanzará cerca de 10 millones en el año 2030, y que sus costes se habrán triplicado con respecto a 2010. Además se calculan en 76 millones los pacientes que viven con factores de riesgo para desarrollar IC (Estadio A de IC) .

Como queda dicho la IC es una enfermedad de la edad avanzada estimándose que por cada década de vida se dobla la incidencia hasta alcanzar al 2-3\% en pacientes >85 años. En mayores de 80 años la prevalencia puede alcanzar hasta el $10 \%$ de la población. En cualquier caso, e independientemente de la edad el riesgo durante la vida de desarrollar IC es de aproximadamente $20 \%$ para todas aquellas personas $>40$ años ${ }^{7}$.

\section{La situación en España}

España no va a la zaga en este proceso. En un estudio de base poblacional realizado en Asturias en 1996, con una muestra bastante limitada, se estimó una prevalencia global de ICC del $4,9 \%$, con una acusada variación etaria $(<1 \%$ en $<50$ años y $18 \%$ en $>80$ años) $)^{8}$.

Una estimación para 2005, utilizando los datos del estudio PRICE realizado en el ámbito ambulatorio, indica que pueden existir en España más de 1,25 millones de personas > 45 años con

Tabla 1. La magnitud mundial de la epidemia de insuficiencia cardiaca

\begin{tabular}{|c|c|}
\hline MUNDIAL: & 23 millones, Incidencia: 5,7 millones/año \\
\hline EUROPA: & 15 millones \\
\hline JAPÓN: & 2,4 millones \\
\hline AUSTRALIA: & 0,3 millones \\
\hline AFRICA: & 3-7\% de ingresos hospitalarios \\
\hline EEUU: & $\begin{array}{l}6,6 \text { millones >18 años, estimados 9,6 millones (2030) } \\
670.000 \text { nuevos (>45 años), } 281.437 † \text { (2008) } \\
\text { 1.094.000 altas, 3,041 millones visitas anuales } \\
39,2 \text { millardos } \$ \text { (2010); estimados } 97,0 \text { (2030) }\end{array}$ \\
\hline
\end{tabular}

Ref.: Global burden of disease, 2004 update. WHO, 2008. Roger VL et al. Heart Disease and Stroke Statistics. 2012 Update. A Report From the American Heart Association. Circulation. 2012;125:e2-e220. Mosterd A, Hoes AW. Heart 2007;93:1137-46. Damasceno A et al. JACC 2007;50:688-93. Mayosi BM. Heart 2007;93:1176-83. este proceso (6,8\% de prevalencia en adultos de esta edad) $)^{9}$. La prevalencia en $>75$ años alcanza el 16\%. Significativamente, en este estudio el $85 \%$ de casos incluidos estaban ya diagnosticados previamente de IC (por hospitalización previa o tras acudir a Urgencias) lo cual indica que en pacientes ambulatorios con IC la aparición de novo es sólo de un 15\% y que el resto de pacientes ambulatorios ya ha consumido recursos importantes del sistema sanitario.

Según los últimos datos disponibles de hospitalización por IC en 2010 se produjeron 113726 altas hospitalarias (Ministerio de Sanidad y Política social, Grupos Relacionados de Diagnósticos: GRD 127 y 544) $)^{10}$. Esto supone un $60 \%$ de incremento desde el año 2000, y se calculan para al año 2020 más de 145.000 altas $^{11}$. El coste hospitalario calculado en 2010 fue de 473 millones $€$ ( $2,6 \%$ del gasto hospitalario), pero su coste real, incluyendo seguimiento extrahospitalario y de cuidadores, se estima en 4-5 veces superior, alcanzando pues cerca de 2500 millones $€$ anuales (3,8\% del gasto sanitario global) ${ }^{12}$. Dos de cada 3 pacientes ingresados con IC son $>75$ años. La mortalidad hospitalaria en 2010 se situó en el 10,4\%10. Las defunciones totales con un diagnóstico de IC en 2010 sobrepasaron las 16000 ( $4^{a}$ causa de mortalidad), de las cuales aproximadamente el $60 \%$ se registraron en el hospital ${ }^{13}$ (Tabla 2).

Según Registros disponibles de seguimiento extrahospitalario tras el ingreso hospitalario por IC en Galicia la mortalidad anual en aquellos pacientes ambulatorios que sobreviven al alta hospitalaria es del 8\% a los 6 meses, y por tanto por tanto, en torno al $16 \%$ de mortalidad anual)(estudio GALICAP ${ }^{14}$ ). En otros estudios recientes comunicados en nuestra Comunidad la mortalidad a los 5 años es del 44\% (Registro EPICOUR ${ }^{15}$ ). En un estudio publicado en España con seguimiento a los 10 años tras la hospitalización por caso incidente esta es cercana al $60 \%{ }^{16}$. Por comparación en EEUU se han publicado cifras

Tabla 2. Situación actual de la insuficiencia cardiaca en España

\begin{tabular}{|l|l|}
\hline Prevalencia (estudio PRICE, 2005): & 1,25 millones (6,8\% >45 años) \\
\hline Hospitalizaciones: & 113.726 altas, EM: 9,0 días \\
\hline Mortalidad hospitalaria: & $10,4 \%$ (11844†) \\
\hline Mortalidad total: & $16.025 \dagger$ (4 causa muerte) \\
\hline Costes hospitalarios: & $\begin{array}{l}\approx 483 \text { millones } € \\
(2,9 \% \text { costes hospital) }\end{array}$ \\
\hline Costes reales: & $\begin{array}{l}\approx 2.270 \text { millones } € \\
\text { (hospital, ambulatorios, } \\
\text { farmacia, cuidadores) }\end{array}$ \\
\hline Estimación 2020: & $>145.000$ altas anuales \\
\hline
\end{tabular}

Ref.: Anguita M et al. Estudio PRICE. Rev Esp Cardiol 2008; 61: 1041-9. NORMA ESTATAL DE LOS AP GRD V23. Año 2010. Ministerio Sanidad y Política Social, CMBD, 2010. Montes J. Arte y riesgo cardiovascular. Madrid: S\&H Medical Science Service, 2010, p. 144 (actualizado 2012 en INE y Ministerio Sanidad y Política social, 2012). 
de mortalidad a 5 años para la década 2000 del 78,5\%, aunque últimamente parece comunicarse mayores tasas de supervivencia, aunque atemperadas por la mayor edad de hospitalización y las comorbilidades asociadas ${ }^{17}$. A lo largo de la historia se observa un desplazamiento de las características de los pacientes y etiología de la IC. Así estos son cada vez más ancianos (en España la mediana de pacientes ingresados con IC es de 78 años) y la etiología isquémica posinfarto tiende a ser sustituida en el anciano por la enfermedad hipertensiva 0 desarrollada años después posinfarto. En paciente ancianos, el $60-70 \%$ de los pacientes presentan insuficiencia cardiaca con FE preservada $(>45-50 \%)^{18}$.

\section{La situación en Galicia}

Galicia, está a la cabeza de CCAA con mayor tasa bruta de mortalidad presenta por insuficiencia cardiaca, aunque cae al $5^{\circ}$ puesto en las tasas ajustadas por edad (fig. 1). Este dato es parcialmente atribuible al mayor envejecimiento de la población gallega (22\% >65 años frente al 17\% nacional, INE 2010) y coherente con el hallazgo de que la edad de defunción por dicha entidad en Galicia sobrepasa en 2 años a las de la media nacional o en 4 a la de otras Autonomías como Andalucía con la mayor mortalidad (Fig. 1) ${ }^{19}$. En cuanto a ingresos hospitalarios Galicia está por encima de la media española en cuanto a tasas de hospitalización por IC [Año 2010: Tasa por 100000 h: 240 (Galicia) vs. 233 (España) $]^{20}$. Ello hace que esta entidad, que constituye la $3^{\text {a }}$ causa de mortalidad en esta comunidad, constituya un importante problema, tanto asistencial como económico. Estimaciones recientes, por ejemplo, han situado los gastos totales asistenciales de la IC en Galicia -intra y extrahospitalarios, además de los domiciliarios para aquellos pacientes hospitalizados en 2004- en 173 millones $€(0,64 \%$ del presupuesto global del SERGAS) $)^{12}$. Estos gastos son pues $4-5$ veces mayores que los ocasionados por hospitalizaciones (35 millones $€$ ). La estancia media hospitalaria en Galicia (9,8 días) es ligeramente superior a la del SNS [Encuesta de morbilidad hospitalaria (EMH-2010): 9,0 días] $]^{20}$ y superponible a la de registros multicéntricos hospitalarios (p. ej. SEMI-IC: 10,2 días) ${ }^{21}$. En cuanto a la mortalidad intrahospitalaria la de Galicia está en torno a 10,4\%, también superponible a la del SNS [CIE 9MC 428 (insuficiencia cardiaca): 107347 altas, mortalidad: $10,1 \%)^{20}$.

Figura 1. Diferencias geográficas en la mortalidad por IC en España. Matices sobre la paradoja española

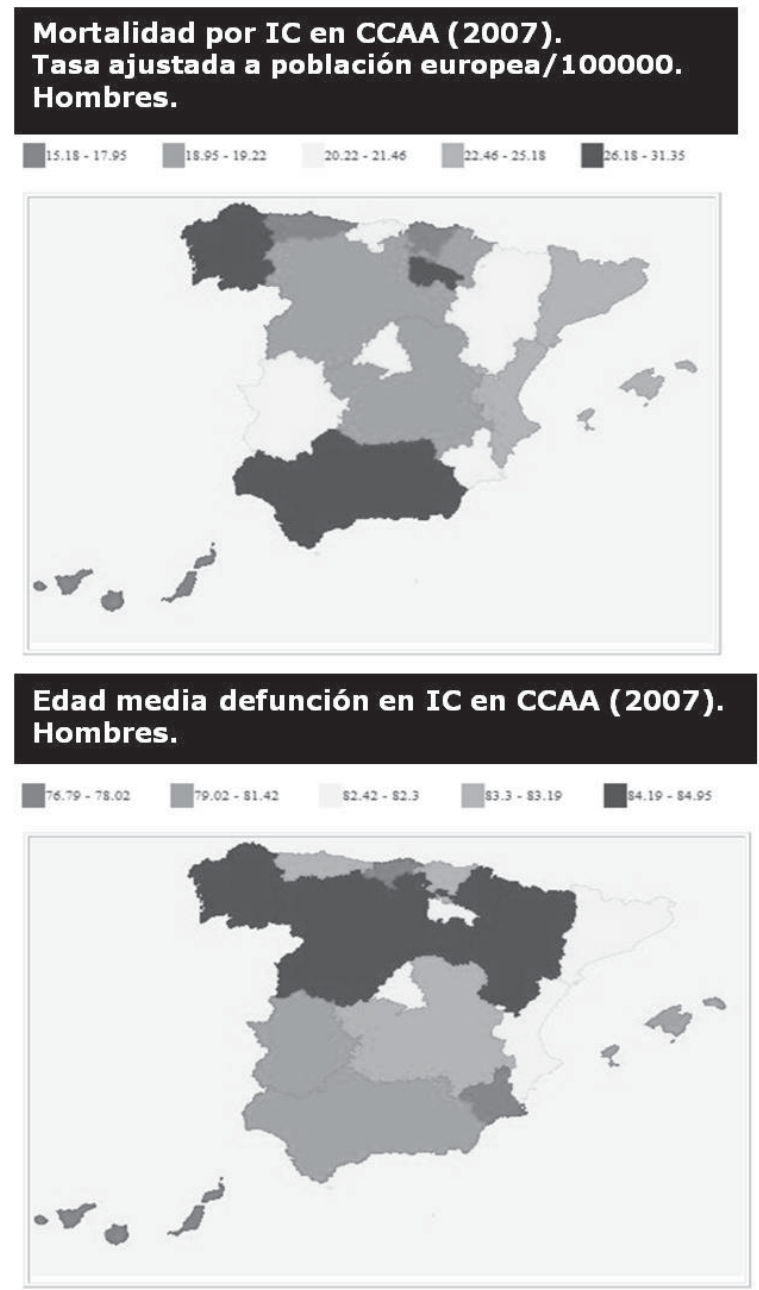

Mortalidad por IC en CCAA (2007) Tasa ajustada a población europea/100000. Mujeres.

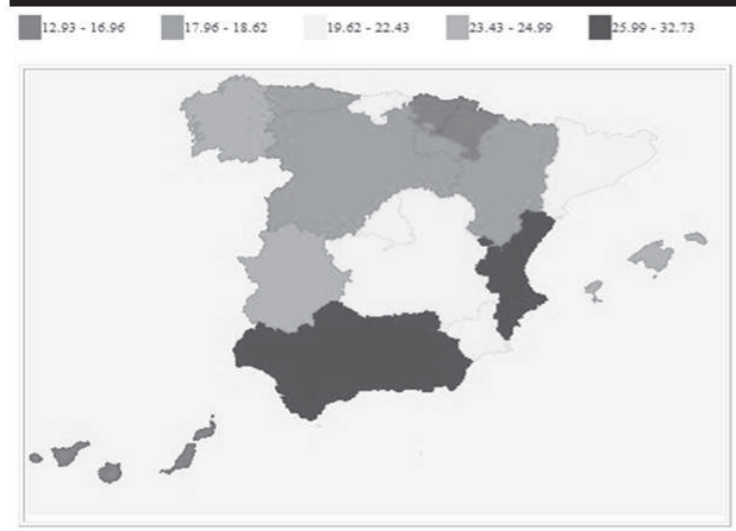

Edad media defunción en IC en CCAA (2007) Mujeres.

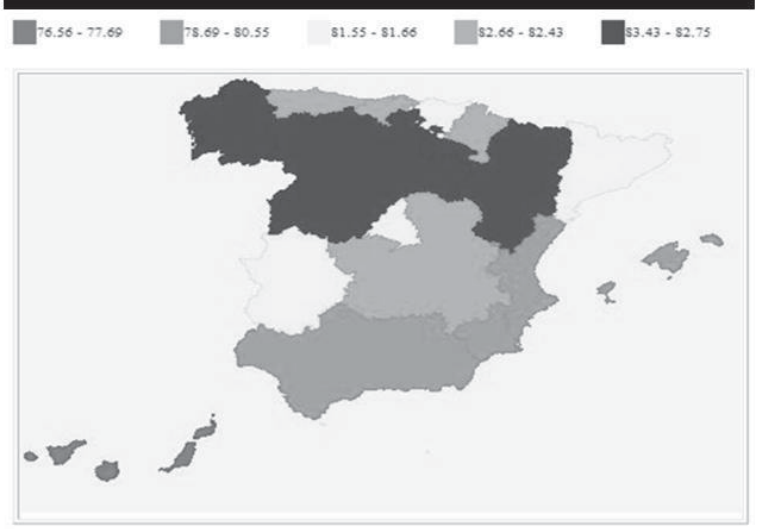




\section{Estudios de IC en diferentes ámbitos asistenciales de Galicia}

En los últimos años se han realizado diversos estudios en pacientes con IC hospitalizados en Galicia con el fin de perfilar sus características, con frecuencia procedentes de un único centro. Para intentar evitar dichos sesgos locales, en este análisis, que actualiza y complementa un estudio nuestro anterior ${ }^{22}$, se revisan aquellos estudios multicéntricos gallegos o bien aquellos que, aunque procedentes de un único centro, hubieran reclutado $>350$ pacientes y seguidos durante un periodo prolongado de tiempo. Se estudiaron y compararon aquellas características demográficas que aparecieran reflejadas en al menos 6 de los 7 estudios, y cuyas características se describen a continuación (por orden cronológico de comunicación 0 publicación de resultados):

Estudio INCARGAL (Insuficiencia cardiaca en Galicia)23. Estudio descriptivo trasversal multicéntrico durante 1999 de 951 pacientes ingresados en Servicios de Medicina Interna y/o Geriatría (587) o Cardiología (364) de 14 hospitales públicos.

Estudio del Servicio de Cardiología del Hospital Clínico Universitario de Santiago (Card-CHUS) ${ }^{24}$. Estudio prospectivo longitudinal de 1252 pacientes ingresados en dicho servicio durante un periodo de 12 años (1991-2002).

Estudio GALICAP (Estudio Gallego de Insuficiencia Cardiaca en Atención Primaria) ${ }^{25}$. Estudio descriptivo transversal multicéntrico de 1195 pacientes ingresados por IC en 2004 y seguidos posteriormente por 149 médicos en Atención Primaria (media de 8 pacientes por médico).

Proyecto GALICEP (Epidemiología de Insuficiencia Cardiaca en Galicia ${ }^{26}$. Estudio descriptivo transversal multicéntrico de 25487 altas de pacientes con IC residentes en Galicia (media de 6372 altas anuales) procedentes del Conjunto Mínimo Básico de Datos al Alta hospitalaria (CMBDAH) de todos los hospitales del SERGAS. Se estudiaron los Grupos Relacionados por el Diagnóstico (GRD) específicos de IC (127 y 544) durante el periodo 2002-2005.
Estudio EPICOUR (Estudio Prospectivo de IC en Ourense) ${ }^{15}$. Cohorte de pacientes seguidos tras hospitalización hospitalaria en Ourense (periodo 1999-2002) y de los que de 2387 pacientes con ecocardiograma realizado se eligieron aleatoriamente 384 con mediana de seguimiento de 58 meses.

Estudio SOGAMI-IC27. Características epidemiológicas de una muestra de pacientes hospitalizados en el año 2006 con diagnóstico principal de IC (Grupos de Diagnóstico Relacionados, GDR: 127 y 544) en los Servicios de Medicina Interna de 9 hospitales de Galicia. Estos Servicios fueron responsables del $74 \%$ de las altas por IC (4848/6569) y los hospitales participantes fueron responsables a su vez del $81,4 \%$ de las altas por IC y el $76,2 \%$ de las altas globales por cualquier patología en Galicia. Estudio de COHORTES DE LUGO28. Estudio de cohortes prospectivo de seguimiento que incluye 456 pacientes ingresados con ICC en el Servicio de e Medicina Interna del Hospital de Lugo desde enero 2006-abril 2009 y seguidos en una consulta monográfica de IC (96 pacientes) o en una consulta general.

\section{Resultados}

Dado que en este análisis se estudiaron los rasgos demográficos comunes a los estudios, se excluyeron los datos de interés aportados por los estudios individuales (p. ej. comorbilidad, situación familiar, etc.) pero no disponibles en todos ellos. En la tabla 3 se reflejan las características comunes de los estudios analizados. A pesar de la heterogeneidad de la metodología de inclusión y de la comunicación de resultados, pueden extraerse de estos estudios algunas conclusiones razonadas. Los pacientes ingresados en Servicios de Cardiología son de menor edad (p. ej. en el estudio INCARGAL -no mostrado en tabla- la edad media de ingresados en Cardiología fue de 73 años, frente a 77 de Medicina Interna/Geriatría, y en el estudio Card-CHUS la media de ingreso fue de 64 años frente a 79 en el estudio SOGAMI-IC. Como se ha comprobado en anteriores ocasiones, los pacientes que ingresan en los servicios de Cardiología son más jóvenes, con mayor frecuencia varones, con cardiopatía isquémica como etiología predominante y mayor proporción de pacientes con FEVI deprimida.

Tabla 3. La IC en diversos estudios en Galicia

\begin{tabular}{|l|c|c|c|c|c|c|c|}
\hline & INCARGAL & Card-CHUS & GALICAP & GALICEP & EPICOUR & SOGAMI-IC & Lugo-IC \\
\hline Año publicación & 2003 & 2005 & 2007 & 2010 & 2010 & 2011 & 2012 \\
\hline No $^{0}$ & 951 & 1252 & 1195 & 25487 & 384 & 432 & 456 \\
\hline Edad media & 76 & 64 & 76 & -- & 75 & 79 & 79 \\
\hline$>75$ años (\%) & 75 & 33 & $79^{\mathrm{a}}$ & 66 & $73^{\mathrm{a}}$ & 64 & \\
\hline Mujeres (\%) & 52 & 37 & 52 & 59 & 47 & 53 & 51 \\
\hline HTA(antecedente) & 59 & 55 & 82 & -- & 60 & & 69 \\
\hline HTA(etiología) & -- & & 36 & 20 & & 47 & 27 \\
\hline Días estancia & 11 & 14 & -- & 10 & & & \\
\hline Mortalidad & 6,8 & $16^{\mathrm{b}}$ & $16^{\mathrm{b}}$ & 10,2 & $53^{\mathrm{c}}$ & 9,3 & $28 \% \%^{\mathrm{b}}$ \\
\hline
\end{tabular}




\section{Discusión}

Aunque ciertos análisis indican que las diferencias por sexo y edad se están reduciendo entre especialidades al menos en los ingresos hospitalarios ${ }^{29}$, los estudios publicados en el ámbito ambulatorio en España, indican que estas discrepancias en la presentación hospitalaria se mantienen también en la asistencia ambulatoria. Así en el estudio BADAPIC (3909 pacientes en consultas ambulatorias de Cardiología) la edad media fue de 66 años, sólo un tercio mujeres y el 40\% presentaba una etiología isquémica ${ }^{30}$. El estudio EPISERVE ${ }^{31}$ que comparó las características de pacientes ambulatorios en consultas de Atención Primaria (778) pacientes), Cardiología (777) y Medicina interna (694) encontró edades medias de 76, 66 y 75 años, en tales especialidades, respectivamente. En Asistencia Primaria (AP), por el contrario, y de forma parecida a lo que ocurre en Medicina Interna (Registros SEMI-IC y RICA) 32,33, Ios pacientes son de edad más avanzada, en mayor proporción mujeres y fundamentalmente hipertensos. En este ámbito asistencial al estudio gallego GALICAP (1195 pacientes, edad: 76 años, 52\% mujeres) deben añadirse $p$. ej. Ios nacionales CARDIOTENS (asistencia ambulatoria cardiológica y de AP, 1420 pacientes, edad: 71 años, 53\% mujeres) ${ }^{34}$, y CARDIOPRES (asistencia en AP, 847 pacientes, edad: 73 años, 49,5\% mujeres) $)^{35}$.

Todo lo anterior es pertinente porque los pacientes que ingresan en los hospitales presentan una destacada comorbilidad, que dificultará el manejo durante el ingreso, pero también durante el régimen ambulatorio. En la misma línea de subrayar las características reales de estos pacientes ingresados en IC, y de la alta prevalencia de la IC que motiva con frecuencia su seguimiento por diversas especialidades, nos parece interesante aquí reseñar una comparación realizada en el área sanitaria de Vigo sobre los Servicios que cuantitativamente son principales responsables del manejo de la IC: Medicina Interna (MI), Geriatría y Hospitalización a Domicilio (HAD0)(Tabla 4) ${ }^{36}$. Tomando como referencia a Ml, en general los pacientes en HADO y Geriatría fueron de mayor edad, más frecuentemente mujeres, con peor capacidad funcional y con mayor polimedicación. Ello se tradujo en la menor supervivencia en los dos últimos servicios, especialmente HADO. La etiología de la cardiopatía no difirió significativamente siendo la más frecuente la hipertensiva. Se comprueba la alta morbimortalidad de la IC, con independencia del Servicio responsable de su atención. Ello se relaciona con la avanzada edad y comorbilidad. Son necesarios protocolos de coordinación y seguimiento entre dichas formas de asistencia para optimizar la atención a dichos pacientes, máxime cuando las características orográficas de Galicia indican que en ella abundan los núcleos de población escasa y de difícil acceso geográfico. Por ello si bien la mortalidad de los pacientes con IC atendidos en HADO es mayor que la de pacientes en hospitalización convencional, debido al mayor deterioro, mayor grado de fragilidad y dependencia y dificultades de acceso hospita- lario esta modalidad asistencial o el desarrollo de unidades de pacientes pluripatológicos o similares pueden constituirse en alternativas eficientes de asistencia.

Es el mismo sentido de resaltar esta dificultad asistencial en Galicia puede observarse en la Fig. 2 [hospitalización por IC en >65 años por áreas sanitarias en el periodo 2002-2005 (Estudio GALICEP ${ }^{25}$ )] la marcada heterogeneidad en cuanto a esta hospitalización entre distintas áreas sanitarias, que ha sido observado incluso dentro de la misma área geográfica ${ }^{37}$, siendo la provincia de Pontevedra la de mayor índice de hospitalización. Con el fin de detectar posibles "bolsas" de prevalencia incrementada de IC en ciertas áreas se realizó un estudio pormenorizado de las mismas, estudiándose todos aquellos municipios con población $>1500$ h mayores de 65 años y más de 12 casos/año de IC (lo que corresponde al menos a 1 caso mensual de hospitalización). Con esta metodología es muy evidente la dispersión de la población gallega pues son numerosos los municipios, (sobre todo en Lugo y Ourense) cuya población es menor de 1500 h. >65 años. Respecto al trienio precedente 1999-2001 en el trienio 2002-04 se produjo un aumento de un $18 \%$ de las hospitalizaciones por IC. Aunque esto puede deberse a variadas causas (disponibilidad de unidades asistenciales ambulatorias o de HADO, problemática

Tabla 4. Características de los pacientes admitidos en Medicina interna, Hospitalización a domicilio (HADO) y Geriatría en el área sanitaria del Hospital Meixoeiro (Vigo)

\begin{tabular}{|c|c|c|c|}
\hline SERVICIO & M. Interna & HADO & Geriatría \\
\hline Periodo & 2006 & 2002-04 & $2005 / 2007$ \\
\hline № (\% altas) & $51(10 \%)$ & $118(10 \%)$ & $110(12 \%)$ \\
\hline Edad & $78 \pm 8$ & $81 \pm 7$ & $86 \pm 4$ \\
\hline Mujeres & 57 & 54 & 70 \\
\hline I. física & & 83 & 70 \\
\hline NYHA III-IV & 76 & 86 & 58 \\
\hline HTA & 53 & 47 & 66 \\
\hline EPOC & 37 & 37 & 38 \\
\hline DM & 33 & 26 & 27 \\
\hline $\begin{array}{l}\text { Enfermedades } \\
\text { neurológicas }\end{array}$ & 24 & 19 & \\
\hline Insuficiencia renal & 33 & 47 & 28 \\
\hline FEVI (>45) & 71 & $(<10 \%$ estudios $)$ & 60 \\
\hline Días de estancia $( \pm \mathrm{DS})$ & $11,8 \pm 7,2$ & $17,0 \pm 14,4$ & 10,6 \\
\hline FA & 51 & 42 & 66 \\
\hline No fármacos & $7,2 \pm 2,2$ & $8,4 \pm 3,0$ & 7,8 \\
\hline Supervivencia al mes & 86 & 81 & 86 \\
\hline
\end{tabular}


Fig. 2. Tasa de altas hospitalarias por IC en las áreas sanitarias hospitalarias de Galicia (Periodo 2002-2005) (Expresadas como \%o en pacientes mayores de 65 años)

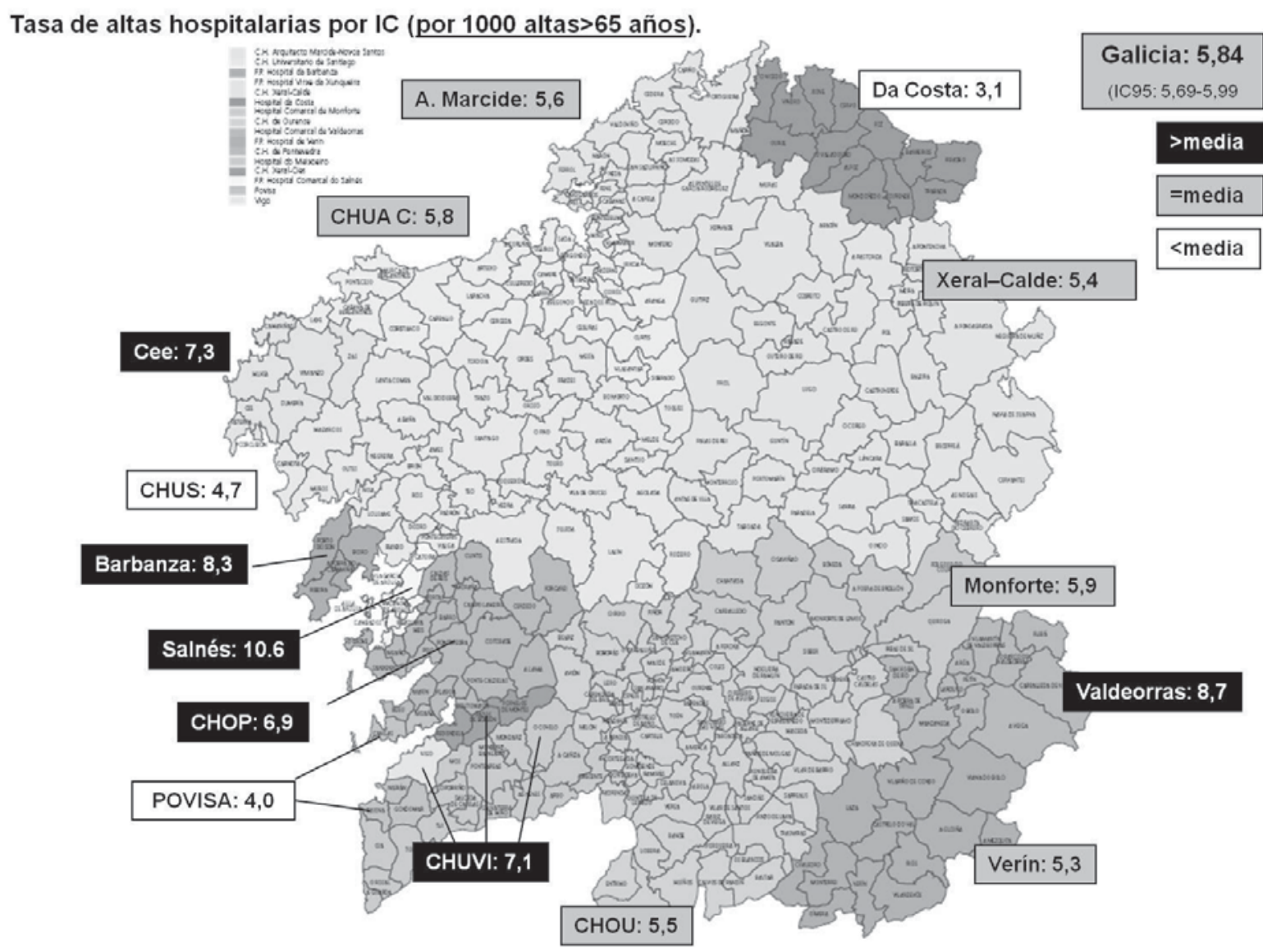

accesibilidad geográfica y por tanto posibles dificultades en las altas hospitalarias, el estudio GALICAP en AP encontró cierta relación entre disminución de hospitalización y mejor control de la HTA, observación que por otra parte está en concordancia con los datos demográficos de hospitalizaciones en otros países $^{14}$. Actualmente En cualquier caso deben tomarse las medidas asistenciales pertinentes para garantizar una asistencia adecuada.

La HTA se recoge como antecedente en la mayoría de los pacientes, aunque la adscripción etiológica de la IC a la HTA se consigna en menor proporción de los casos. La comorbilidad asociada es muy importante y en este sentido cabe calificar a la IC como una enfermedad con repercusión multisistémica. En la tabla 4 se representa la comorbilidad hallada en los cerca de 7000 ingresos anuales por IC >35 años en el Servicio Gallego de Salud (SERGAS) ${ }^{38}$. Es de reseñar que en este el $74 \%$ de las altas se produjeron en Medicina Interna, el 16\% en Cardiología y el resto en otras especialidades (Geriatría, Neumología, Nefrología, etc.). Como se comentaba anteriormente en Galicia apenas hubo diferencias relevantes en las edades de ingreso según los Servicios [Edad media (años): Global: 78,2; Cardiología: 78,0; Medicina Interna: 78,2; Geriatría: 80,4 , Resto: 78,1 ) El $70 \%$ de las pacientes eran $>75$ años y el $80 \%$ de la letalidad se produjo en este grupo etario.
La mortalidad durante un ingreso por IC es variable pero en Galicia, al igual que en España se mantiene en torno al 10\% de los ingresos por IC, y esta no ha variado significativamente en los últimos 15 años $^{39}$. Se evidencia que la mayor parte de los pacientes que ingresan por IC en Galicia presentan antecedentes de HTA, aunque la atribución etiológica de la IC a HTA se refleja en un número mucho menor de casos.

Tabla 4. Comorbilidad en pacientes con IC >35 años ingresados en el SERGAS (2006)

\begin{tabular}{|l|c|c|}
\hline & $\mathrm{N}^{0}$ & $\%$ \\
\hline Episodios & 6569 & \\
\hline Edad (DS) & $78,2(9,9)$ & \\
\hline Mujeres & 3456 & $52,6 \%$ \\
\hline HTA (etiología) & 1378 & $21,0 \%$ \\
\hline HTA (antecedentes) & 3096 & $47,1 \%$ \\
\hline Fibrilación auricular & 3284 & $50,0 \%$ \\
\hline EPOC & 2259 & $34,4 \%$ \\
\hline Enfermedad cerebro-vascular & 377 & $5,7 \%$ \\
\hline Demencia & 252 & $3,8 \%$ \\
\hline Nefropatía & 1038 & $15,8 \%$ \\
\hline Diabetes & 2283 & $34,8 \%$ \\
\hline Letalidad & 688 & $10,5 \%$ \\
\hline
\end{tabular}


Por otra parte se observa una creciente mejor utilización de los recursos diagnósticos en esta entidad y por ej. la realización de ecocardiograma en Servicios de Medicina Interna que era sólo de un 41\% (Estudio INCARGAL, 200322) ha pasado al 48\% (Estudio EPICOUR $\left.{ }^{15}, 2010\right), 67 \%$ (Estudio GALICAP, 2009 ${ }^{14}$ ) 0 al 69\% [Estudio SOGAMI-IC 201026, datos de Hospital Meixoeiro)]. Tales porcentajes son similares a la de registros realizados en periodos semejantes en Registros nacionales (Estudio SEMI-IC: 2145 pacientes, ecocardiograma: $72 \%)^{21}$, si bien todavía algo alejados de otros registros europeos (EuroHeart Failure Survey Il: 3580 pacientes, ecocardiograma: 89\% ${ }^{40}$.

En conclusión, pues, los pacientes que ingresan por IC en Galicia son muy ancianos y con elevada comorbilidad por lo que esta entidad debe considerarse como una enfermedad multisistémica. Es preciso pues un enfoque integral de dicha entidad para su manejo adecuado. Sería deseable que todos los estamentos implicados en el cuidado de la IC pudieran recibir formación específica en el cuidado de pacientes pluripatológicos.

\section{Bibliografía}

1. McMurray JJV, Adamopoulos S, Anker SD et al. ESC Guidelines for the diagnosis and treatment of acute and chronic heart failure 2012: The Task Force for the Diagnosis and Treatment of Acute and Chronic Heart Failure 2012 of the European Society of Cardiology. Developed in collaboration with the Heart Failure Association (HFA) of the ESC. Eur J Heart Fail. 2012; 14(8):803-69.

2. National Clinical Guideline Centre (2010). Chronic heart failure: the management of chronic heart failure in adults in primary and secondary care. London: National Clinical Guideline Centre. Available from: http://guidance.nice.org.uk/CG108/Guidance/pdf/English.

3. Mosterd A, Hoes AW. Clinical epidemiology of heart failure. Heart 2007;93:1137-1146.

4. Krum H, Abraham WT. Heart failure. Lancet 2009; 373: 941-55.

5. McDonald K. Prevalencia de la insuficiencia cardiaca: un reflejo de lo bueno y lo malo de la asistencia cardiovascular moderna. Rev Esp Cardiol. 2008;6:1010-2

6. Roger VL, Go AS, Donald M. Lloyd-Jones DM et al. Heart Disease and Stroke Statistics. 2012 Update. A Report from the American Heart Association. Circulation. 2012; 125:e2-e220.

7. Lloyd-Jones DM, Larson MG, Leip EP et al. Lifetime risk for developing congestive heart failure: the Framingham Heart Study. Circulation 2002; 106: 3068-72.

8. Cortina A, Reguero J, Segovia E et al. Prevalence of heart failure in Spain (a region in the north of Spain). Am J Cardiol. 2001; 87: 1417-9.

9. Anguita Sánchez M, Crespo Leiro MG; de Teresa Galván E et al. Prevalencia de la insuficiencia cardiaca en la población general española mayor de 45 años. Estudio PRICE. Rev Esp Cardiol. 2008; 61:1041-9.

10. Ministerio de Sanidad, Política Social e Igualdad. Instituto de Información Sanitaria. Registro de altas - CMBD. http://pestadistico.msc.es [Consultados, 02-09-2012].

11. Montes-Santiago J. El riesgo cardiovascular en el arte. Madrid: S\&H Medical Science Service 2010, p. 144.

12. Montes-Santiago J, Rey García G, Mediero Domínguez A. Economic burden of heart failure in Galicia (Northwest of Spain): The GALICEP-costes study. European Federation of Internal Medicine, $6^{\text {th }}$ Congress. Lisbon 2007(May 23-26); Abstract book: P-49-07.

13. Instituto Nacional de Estadística (INE). Defunciones según la causa de muerte. Disponible en http://www.ine.es/inebase/cgi/axi. Principales causas de muerte por comunidades autónomas de residencia (2010). Encuesta de Morbilidad Hospitalaria, 2010. Disponible en: http://www.ine.es/jaxi/tabla. [Consultados, 02-09-2012].

14. Otero-Raviña F, Grigorian-Shamagian L, Fransi-Galiana L et al.; GALICAP Study researchers. Morbidity and mortality among heart failure patients in Galicia, N.W. Spain: the GALICAP Study. Int J Cardiol. 2009; 136:56-63.

15. J. López Castro J, Almazán Ortega R, Pérez de Juan Romero M, González Juanatey $J R$. Factores pronósticos de mortalidad de la insuficiencia cardíaca en una cohorte del noroeste de España. Estudio EPICOUR. Rev Clin Esp. 2010; 210:438-47.

16. Quirós López R, García Alegría J, Martín Escalante MD et al. Prognostic factors and long-term survival after initial diagnosis of heart failure]. Med Clin (Barc). 2012 May 19;138(14):602-8.

17. Goldberg RJ, Ciampa J, Lessard D, Meyer TH, Spencer FA. Long-term survival after heart failure. A contemporary population-based perspective. Arch Intern Med. 2007;167: 490-96.
18. Montes- Santiago J. Insuficiencia cardiaca con fracción de eyección preservada. Rev Clin Esp 209 (Supl. 2): 3-10.

19. Montes-Santiago J, Álvarez J, Rey G et al. Diferencias geográficas en la mortalidad por insuficiencia cardíaca en España. Matizando la paradoja española. Rev Clin Esp 2010 (suppl esp); 210: 250.

20. INE. Encuesta de morbilidad hospitalaria, 2010. Disponible en: http://www.ine.es/ jaxi/tabla.do (Consultado 4/09/2012).

21. Grupo de Trabajo de Insuficiencia Cardíaca de la Sociedad Española de Medicina Interna (SEMI). La insuficiencia cardíaca en los servicios de medicina interna (estudio SEMI-IC). Medicina Clínica (Barc) 2002; 118: 605-610.

22. Montes-Santiago J, Lado Castro-Rial M, Rey García G et al. Demografía de la insuficiencia cardiaca en Galicia. Análisis de diferentes poblaciones y ámbitos asistenciaIes Galicia Clin 2008; 69: 7-10.

23. García Castelo A, Muñiz García J, Sesma Sánchez P, Castro Beiras A. Utilización de recursos diagnósticos y terapéuticos en pacientes ingresados por insuficiencia cardiaca: influencia del servicio de de ingreso (estudio INCARGAL). Rev Esp Cardiol. 2003; 56:49-56.

24. Varela-Román A, Grigorian Shamagian L, Barge E et al. Heart failure in patients with preserved and deteriorated left ventricular ejection fraction: Iong term prognosis. Heart. 2005; 91:489-94.

25. Otero-Raviña F, Grigorian-Shamagian L, Fransi-Galiana $L$ et al. Estudio gallego de insuficiencia cardiaca en atención primaria (estudio GALICAP). Rev Esp Cardiol. 2007; 60:373-83.

26. Montes-Santiago J, Rey-García G, Mediero-Domínguez A. El proyecto GALICEP: Epidemiología de la insuficiencia cardiaca en el anciano en Galicia. Variaciones geográficas provinciales. Rev Clin Esp 2006;206 (Supl. 3):160.

27. Montes J, Chouciño T, Gómez-Guiguirey A et al. Características epidemiológicas de los pacientes ingresados por insuficiencia cardíaca en medicina interna en Galicia: estudio SOGAMI-IC. Rev Clin Esp 2010 (suppl esp); 210: 241.

28. Cerqueiro González JM, Abeledo Vázquez C, De la Fuente Sánchez S, Casariego Vales E. Influencia sobre la hospitalización de una consulta monográfica para pacientes con insuficiencia cardiaca dirigida porinternistas. Estudio de cohortes. Galicia Clin 2012; 73 (Supl.1): S27-S29.

29. Permanyer Miralda G, Soriano N, Brotons C et al. Características basales y determinantes de la evolución en pacientes ingresados por insuficiencia cardiaca en un hospital general. Rev Esp Cardiol. 2002;55:571-8.

30. Anguita Sánchez M, Investigadores del Registro BADAPIC. Características clínicas, tratamiento y morbimortalidad a corto plazo de pacientes con insuficiencia cardiaca controlados en consultas específicas de insuficiencia cardiaca. Resultados del Regiatro BADAPIC. Rev Esp Cardiol. 2004;57:1159-69.

31. González-Juanatey JR, Alegría Ezquerra E, Bertoméu Martínez V et al. Insuficiencia cardiaca en consultas ambulatorias:comorbilidades y actuaciones diagnóstico-terapéuticas por diferentes especialistas. Estudio EPISERVE. Rev Esp Cardiol. 2008; 61:611-9

32. Montero Pérez-Barquero M, Conthe Gutiérrez P, Román Sánchez P, García Alegría J, Forteza-Rey J; Grupo de Trabajo de Insuficiencia Cardiaca de Sociedad Española de Medicina Interna (estudio SEMI-IC). Comorbilidad de los pacientes ingresados por insuficiencia cardiaca en los servicios de medicina interna. Rev Clin Esp. 2010; 210:149-58.

33. Casado J, Montero M, Formiga F et al.; Grupo RICA. Función renal en pacientes con insuficiencia cardiaca. Valor pronóstico. Rev Clin Esp. 2012; 212:119-26.

34. Barrios Alonso V, Peña Pérez G, González Juanatey JR et al. Hipertensión arterial e insuficiencia cardiaca en las consultas de atención primaria y de cardiología en España. Rev Clin Esp. 2003;203:334-42.

35. Rodríguez Roca GC, Barrios Alonso V, Aznar Costa J, et al., en representación de SEMERGEN, de la Sección de Cardiología Clínica y Extrahospitalaria de la Sociedad Española de Cardiología y de los investigadores del estudio CARDIOPRES. Características clínicas de los pacientes diagnosticados de insuficiencia cardiaca crónica asistidos en Atención Primaria. Estudio CARDIOPRES. Rev Clin Esp. 2007;207:337-40.

36. Montes-Santiago J, A. Vilches-Moraga A, Amador-Barciela L, G. Rey-García G. Comparación de las características de pacientes con insuficiencia cardiaca en el sur de Galicia según tres servicios de referencia: Medicina Interna, Geriatría y HADO. Rev Clin Esp 2011 (Espec Cong): 251-2.

37. Montes-Santiago J, Rey-García G, Mediero-Domínguez A. Diferencias geográficas locales en las altas por insuficiencia cardiaca en ancianos en un área de Galicia. Comparación con la hospitalización convencional. Rev Clin Esp. 2006;206 (Supl. 3):152.

38. Montes-Santiago J, Lado M, Fernández C et al. Comorbilidades en la insuficiencia cardiaca hospitalizada en Galicia. Comunicación oral XV Congreso da Sociedade Galega de Hipertensión (SOGATHA), 2008.

39. J Montes-Santiago, J. Álvarez, R. Guijarro, C. San Román, M. Monreal. Evolución y características de la mortalidad hospitalaria por insuficiencia cardíaca en España. Rev Clin Esp 2009; 209 (suppl. esp): 136.

40. Nieminen MS, Brutsaert D, Dickstein $\mathrm{K}$ et al, on behalf of the EuroHeart Survey Investigators. EuroHeart Failure Survey II (EHFS II): a survey on hospitalized acute heart failure patients: description of population. Eur Heart J. 2006 27:2725-36. 